\title{
Perfil epidemiológico e atitudinal de saúde bucal de gestantes assistidas em um Ambulatório Docente Assistencial em Salvador, BA
}

\author{
Epidemiological and attitudinal oral health profile of pregnant women assisted in an \\ educational Ambulatory Care Center at Salvador, BA
}

\author{
Alena Ribeiro Alves Peixoto Medrado ${ }^{1 *}$, Maiana Mendes Nunes², Monah Sampaio Santos², Vanessa \\ Vasconcelos Lessa ${ }^{3}$
}

\begin{abstract}
${ }^{1}$ Doutora em Patologia Humana. UFBA. Professora Adjunto do Departamento de Biointeração. UFBA e da Escola Bahiana de Medicina e Saúde Pública; ${ }^{2}$ Graduada em Odontologia. Escola Bahiana de Medicina e Saúde Pública; ${ }^{3}$ Acadêmica de Odontologia. Escola Bahiana de Medicina e Saúde Pública.
\end{abstract}

\begin{abstract}
Resumo
Introdução: Este trabalho traçou o perfil epidemiológico e atitudinal de gestantes assistidas no Ambulatório Docente Assistencial ADAB, da Escola Bahiana de Medicina e Saúde Pública, em Salvador, BA, entre 2013 e 2014. Objetivo: Conhecer a condição gengival das gestantes e avaliar seus conhecimentos sobre as alterações bucais, sobre verdades e mitos relacionados à saúde bucal e ao atendimento odontológico durante a gravidez e, suas atitudes frente à higiene bucal. Metodologia: Foi realizado um estudo do tipo transversal qualiquantitativo. Exame clínico bucal e entrevista oral foram realizados com uma população de 17 gestantes. A amostra contou com mulheres entre o 1 으 e 9 ㅇ mês de gestação, com idades entre 17 e 36 anos. Resultados: Foram observadas alterações gengivais, a exemplo de sangramento durante a escovação e gengiva edemaciada, sendo que $76,5 \%$ das gestantes apresentaram Índice Gengival > 10\% e 88,3\% apresentaram Índice de Placa > 20\%. Constatou-se um baixo nível de conhecimento em relação aos procedimentos odontológicos que podem ser realizados durante a gravidez e, confirmou-se a relação entre crenças populares e gestação que afastam as gestantes do tratamento odontológico. Conclusão: Neste estudo, verificou-se a necessidade da adoção de medidas educativo-preventivas, com enfoque na orientação e motivação das gestantes, bem como desmistificar o acompanhamento odontológico durante o pré-natal.
\end{abstract}

Palavras-chave: Epidemiologia. Gravidez. Saúde Bucal. Atitude.

\begin{abstract}
Introduction: This study was to establish epidemiological and attitudinal profile of pregnant women assisted at Educational Ambulatory Care Center of Bahiana, at Escola Bahiana de Medicina e Saúde Pública, Salvador, BA, during the period 2013-2014. Purpose: To know the gingival condition of pregnant women and assess their knowledge on oral abnormalities, on truths and myths related to oral health and dental care during pregnancy, and their attitudes towards oral hygiene. Methodology: A study cross-type quantitative quality wasconducted. Oral clinical examination and oral interviews were conducted with a population of 17 pregnant women. The sample consisted of women between the 1st and 9th month of pregnancy, aged between 17 and 36 years. Results: Gingival changes were observed, like bleeding during brushing and swollen gums, with $76.5 \%$ of the patients presented Gingival Index $>10 \%$ and $88.3 \%$ had Plaque Index $>20 \%$. It found a low level of knowledge regarding dental procedures that can be performed during pregnancy. It confirmed the relationship between popular beliefs and pregnancy that keep pregnant women dental treatment. Conclusion: In this study, there was a need to adoption educational-preventive measures, focusing on guidance and motivation of pregnant women, and demystify the dental monitoring during the prenatal.
\end{abstract}

Keywords: Epidemiology. Pregnancy. Oral health. Attitude.

\section{INTRODUÇÃO}

O período gestacional acarreta uma série de mudanças na vida da mulher. Há uma maior preocupação com o estado de saúde geral por parte da gestante. Entretanto, a saúde bucal parece ser relegada a segundo plano e a busca por atendimento e acompanhamento odontológico durante a gravidez, em razão dos diversos mitos e crenças populares existentes, são retardados ao máximo ou nem sequer cogitados.

Correspondência/Corresponding: *Alena R. A. P. Medrado - Endereço: Rua Rodolfo Coelho Cavalcante, № 90, Apto. № 502, Jardim Armação. Salvador, BA - CEP: 41750-166 - Tel: 71 3272-2042/ 98838-0218 - E-mail: alenamedrado@hotmail.com
A experiência da gravidez pode provocar muita ansiedade e receio quando a futura mãe ainda não pôde ter vivido um processo de gravidez no seio familiar e/ou não pôde ter tido contato com um recém-nascido. Ao longo da vida, a mulher vai ouvindo relatos de experiências e histórias que se transmitem sucessivamente de mães para filhas e que se configuram em crenças ou mitos determinantes dos seus comportamentos, gerando receios e medos na sua gravidez.

A baixa percepção de suas necessidades bucais, as crenças populares, o medo e a ansiedade, a falta de interesse, a preguiça, a indiferença, o fato de não gostar de dentista ou de nem pensar em ir ao dentista durante 
a gravidez, a baixa valorização da saúde bucal, a pouca importância atribuída aos dentes, o baixo poder aquisitivo e a dificuldade de acesso aos serviços de saúde pública são alguns exemplos de barreiras ao tratamento odontológico (ALBUQUERQUE; ABEGG; RODRIGUES, 2004).

É cediço que muitas gestantes, apresentando receio de que a execução dos procedimentos pelo cirurgião- dentista venha a causar danos ao bebê, deixam de procurar atendimento odontológico. Alves et al. (2010), observaram que $79,8 \%$ das gestantes participantes do seu estudo não souberam quais os procedimentos podiam ou não ser realizados durante a consulta odontológica. De acordo com o Ministério da Saúde, há um período preferencial para a realização de intervenções clínicas e procedimentos odontológicos que é o segundo trimestre de gravidez. Isso porque durante o primeiro trimestre ocorrem transformações embriológicas, ou seja, é o início da morfodiferenciação fetal. Tomadas radiográficas devem ser evitadas neste período. Já na fase correspondente ao terceiro trimestre, há maior risco de síncope, hipertensão e anemia. É frequente o desconforto na cadeira odontológica, podendo ocorrer hipotensão postural, em decorrência do aumento do tamanho do útero gravídico que causa compressão da veia cava e artéria aorta quando da colocação da gestante em posição supina. No entanto, considerando-se que a manutenção de infecções na cavidade oral da grávida possa trazer maiores prejuízos ao bebê do que o próprio tratamento odontológico em si, deve-se sempre buscar avaliar em cada circunstância o risco frente ao benefício do tratamento (BRASIL, 2010).

A situação de saúde bucal pode desempenhar um papel importante na etiopatogenia de diversas condições durante a gestação, sendo, por isso, extremamente importantes os cuidados com a saúde oral (REIS et al., 2010).

As gestantes representam um grupo populacional de pacientes de risco temporário aos procedimentos odontológicos. As diversas modificações decorrentes da gravidez sejam elas de ordem psíquica, física, hormonal ou nutricional, proporcionam o desenvolvimento de algumas condições desfavoráveis ao meio bucal (VASCONCELOS et al., 2012).

Várias são as alterações que ocorrem na cavidade oral das gestantes, e estas podem ser explicadas pelo desequilíbrio da atividade metabólica ocasionada pela elevação de taxas hormonais. Os níveis elevados de progesterona na gravidez aumentam a permeabilidade e dilatação capilar e, desta forma, aumentam o exsudato gengival. Os níveis elevados de estrogênio, somados ao de progesterona, afetam o grau de ceratinização do epitélio gengival, resultando em menor efetividade da barreira epitelial em mulheres grávidas. Os fatores hormonais que afetam o epitélio e aumentam a permeabilidade vascular podem contribuir para uma resposta exagerada à placa bacteriana durante a gravidez, resultando em gengivite (REIS et al., 2010).

Muitas gestantes acreditam na hipótese de que seus dentes ficam mais fracos e propensos à cárie dentária por perderem minerais para os ossos e dentes do bebê em desenvolvimento (MESQUITA, et al. 2013). Bastiani et al. (2010) encontraram em sua pesquisa que $48,75 \%$ das gestantes acharam que era normal desenvolver cárie dentária durante o período gestacional, uma vez que muitas mães relacionaram que os dentes ficam mais fracos pela transmissão de minerais, como o cálcio, para os dentes do bebê. Apesar do mito existente, deve-se esclarecer que a gravidez não é responsável pelo aparecimento de cáries e nem pela perda de minerais dos dentes da mãe. A cárie é uma doença infectocontagiosa, que depende de fatores primários (microbiota, dieta inadequada, hospedeiro suscetível, tempo) e outros fatores moduladores (idade, sexo, nível socioeconômico) que atuam conjuntamente, por um determinado período, a fim de que a doença possa se estabelecer. Portanto, alterações de hábitos alimentares resultantes do fato de estar grávida; aumento da frequência das refeições; maior exposição do esmalte ao ácido gástrico (proveniente de episódios de vômito); e negligências com a higiene bucal, são fatores que contribuem para o surgimento da doença cárie (REIS, et al. 2010).

Adicionalmente, as gestantes constituem-se um grupo populacional estratégico para a educação em saúde, já que se apresentam mais receptivas à aquisição de novos conhecimentos e hábitos benéficos à saúde do bebê. É essencial que essa educação seja realizada de forma multidisciplinar, visando garantir a introdução de hábitos saudáveis desde o início da gestação. Por essas razões, é de suma importância o engajamento do cirurgião-dentista como agente promotor da saúde dentro de um programa de pré-natal (ROSA et al., 2007).

A execução do presente estudo pode ser justificada pela necessidade de não somente se traçar um perfil epidemiológico, mas também de se enfatizar a relevância da adoção de medidas preventivas na melhoraria da saúde bucal deste segmento populacional. Neste sentido, objetivou-se conhecer a condição gengival em pacientes gestantes, bem como avaliar seus conhecimentos sobre as alterações bucais, suas atitudes frente à higiene bucal, crenças e mitos relacionados à saúde bucal e ao atendimento odontológico, durante a gravidez.

\section{METODOLOGIA}

Foi realizado um estudo epidemiológico do tipo corte transversal qualiquantitativo, conduzido no Ambulatório Docente Assistencial da Bahiana - ADAB, na cidade de Salvador-BA, durante o período de 2013 a 2014. O estudo foi aprovado pelo Comitê de Ética em Pesquisa - CEP da Escola Bahiana de Medicina e Saúde Pública n. 140.993 e pela Comissão Nacional de Ética em Pesquisa - CONEP n. 02494812.3.0000.5544.

Utilizou-se uma amostra por conveniência composta pelas gestantes que estivessem participando do Programa de Pré-Natal e que aceitassem participar da pesquisa, voluntariamente. Foram incluídas gestantes em boas condições de saúde, sem distinção de raça, nível socioeconômico ou escolaridade. Não houve restrição 
quanto ao período de gestação, assim a gestante poderia encontrar-se no primeiro, segundo ou terceiro trimestre de gestação.

A população de estudo constituída por gestantes agendadas para a consulta pré-natal no ADAB-Brotas recebeu esclarecimentos verbais acerca dos objetivos $\mathrm{e}$ procedimentos da pesquisa. Aquelas que aceitaram participar da pesquisa assinaram, em duas vias, o Termo de Consentimento Livre e Esclarecido (TCLE).

Após assinatura do TCLE, duas entrevistadoras aplicaram um questionário referente às condições sociais da gestante, assim como escolaridade, moradia e outros determinantes sociais, bem como um outro questionário inicial que perquiria o conhecimento sobre saúde e higiene bucal, atendimento e acompanhamento odontológico, durante a gravidez. Em continuidade, foram feitas explanações e orientações individualizadas para cada gestante e, posteriormente, aplicação do questionário final com mesmo conteúdo do inicial com fito de avaliar a compreensão acerca do exposto.

As atitudes das gestantes frente aos diversos aspectos de saúde bucal foram avaliadas por meio de dois questionários elaborados pelos pesquisadores, totalizando 36 perguntas. As fichas foram conferidas uma a uma, para averiguar se todos os campos foram devidamente preenchidos; as que apresentaram ausência de resposta foram excluídas. As informações coletadas foram armazenadas por uma única pesquisadora em um banco de dados elaborado especialmente para este fim, utilizando-se o programa Excel para Windows (2007).

No mesmo período, foi realizado o exame intrabucal para determinar a prevalência de lesões bucais e avaliar o Índice de Placa (IP) e Índice Gengival (IG). A presença de sangramento gengival e placa visível foram avaliados de acordo com o índice de Ainamo e Bay (1975), através do qual as superfícies mesial, distal, vestibular e lingual das unidades dentárias presentes foram examinadas. $O$ cálculo foi feito dividindo-se o número de superfícies contendo placa e exibindo sinais de sangramento, pelo número total de superfícies examinadas. Os resultados foram expressos em porcentagem.

O exame clínico foi realizado sob luz ambiente, com a paciente sentada em cadeira comum. Todas as normas de biossegurança foram empregadas com o intuito de minimizar os riscos de contaminação, como o uso de avental, gorro, máscara e luvas descartáveis pelas examinadoras para proteção individual e o emprego dos materiais (espelho bucal e sonda periodontal da OMS) devidamente autoclavados em envelopes individuais. A sonda utilizada apresenta uma ponta esférica com $0,5 \mathrm{~mm}$ de diâmetro, uma faixa preta entre 3,5 e 5,5 mm e anéis com 8,5 e 11,5 $\mathrm{mm}$ de distância da ponta. Ela foi utilizada percorrendo-se sua ponta ativa pelo sulco de todos os dentes presentes em cada gestante, tomando-se o cuidado de mínima pressão da ponta na base do sulco, e colocação da ponta ativa o mais paralelamente possível ao longo eixo dentário.

Os encontros com as gestantes ocorreram antes ou após as consultas do pré-natal com um tempo de duração de aproximadamente 45 minutos cada entrevista. Atividades de sala de espera abordando temas como "alterações hormonais e bucais durante a gravidez" e "higiene íntima", fizeram parte das atividades desenvolvidas durante a pesquisa.

A partir da percepção e das condições de saúde bucal, procurou-se, através de medidas educativo-preventivas, orientar e motivar as gestantes, sujeito fundamental nesse processo de promoção da saúde, em relação aos cuidados com a higiene e saúde oral, bem como desmistificar o acompanhamento odontológico durante o pré-natal.

Os dados foram analisados através de estatística quantitativa descritiva abrangendo valores absolutos e percentuais.

\section{RESULTADOS}

Participaram do estudo 24 gestantes, sendo 19 entrevistadas no ano de 2013 e 5 em 2014. Destas, 7 (29,1\%) não tiveram as fichas devidamente preenchidas, sendo, por isso, excluídas da análise. A amostra final foi composta por 17 (70,9\%) gestantes. A idade da população de estudo variou entre 17 e 36 anos, com média de 29,05 anos.

Dezesseis $(94,1 \%)$ gestantes eram predominantemente adultas (apresentavam 20 anos ou mais) e 17 (100\%) não se autoconsideravam brancas. Nove $(52,9 \%)$ eram solteiras e $8(47,1 \%)$ viviam com parceiros estáveis, ou seja, eram casadas ou viviam em regime de união estável. Mais da metade da amostra (70,5\%) trabalhava fora de casa, 13 (76,5\%) concluíram o 2 o grau e 15 (88,3\%) tinham renda familiar superior a um salário mínimo em valores vigentes ao respectivo ano.

Os resultados relativos ao exame clínico demonstraram que $13(76,5 \%)$ gestantes apresentaram Índice Gengival (IG) superior a 10\% e 15 (88,3\%) apresentaram Índice de Placa (IP) superior a $20 \%$.

Foram analisados 10 itens relativos ao grau de importância dada ao acompanhamento odontológico, ao conhecimento sobre tratamento odontológico e saúde bucal, e crenças e mitos relacionados à assistência odontológica, durante a gravidez, que mostraram uma grande falta de conhecimento por parte das gestantes. Elas estavam desinformadas sobre como prevenir as possíveis alterações bucais que podem ocorrer durante o período gestacional; a maioria das gestantes não se apercebia de que seus problemas bucais poderiam afetar a saúde do futuro bebê. Muitas ainda exibiram crenças e mitos fortemente relacionados à gestação e, embora as gestantes considerassem importante o atendimento odontológico preventivo, o principal motivo de consulta ao dentista ainda era o tratamento curativo.

Os dados referentes à primeira aplicação do questionário demonstraram que $7(41,2 \%)$ participantes da amostra afirmaram que o exame radiográfico é contraindicado na gravidez. Oito (47\%) gestantes afirmaram que não há um período específico da gravidez em que seja melhor a realização de atendimento odontológico. Nove (53\%) participantes acreditaram que não poderiam fazer uso de 
anestésico local e 11 (64,7\%) declararam que não deveriam realizar extração dentária. Dentre as gestantes examinadas, $11(64,7 \%)$ relataram ter observado alguma alteração na mucosa oral durante a gestação, a exemplo de sangramento durante a escovação e gengiva edemaciada. Também foi relatada a percepção de sensibilidade dentária (Figura 1).

Figura 1 - Distribuição numérica das respostas referentes ao conhecimento das gestantes acerca do acompanhamento e tratamento odontológico durante a gravidez, antes da realização da palestra educativa. Ambulatório Docente Assistencial da Bahiana - ADAB, Salvador/BA, 2013 e 2014.

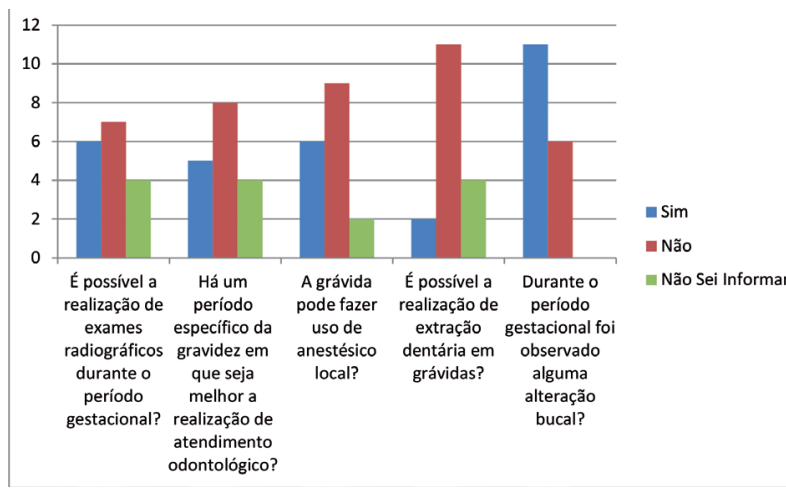

Por outro lado, os resultados relativos às crenças e mitos relacionados à saúde bucal, apresentados na Figura 2 , mostraram que $11(64,7 \%)$ gestantes afirmaram que a gravidez fragiliza os dentes. Nove $(53 \%)$ relataram que a suplementação de flúor é necessária neste período, e 11 $(64,7 \%)$ responderam que o uso de chupeta é prejudicial ao bebê. Quando questionadas sobre a influência que a mudança do hábito alimentar poderia ter sobre a saúde bucal, $6(35,3 \%)$ gestantes afirmaram não haver influência.

Figura 2 - Distribuição numérica das respostas referentes às crenças e mitos relacionados à saúde bucal, antes da realização da palestra educativa. Ambulatório Docente Assistencial da Bahiana - ADAB, Salvador/BA, 2013 e 2014.

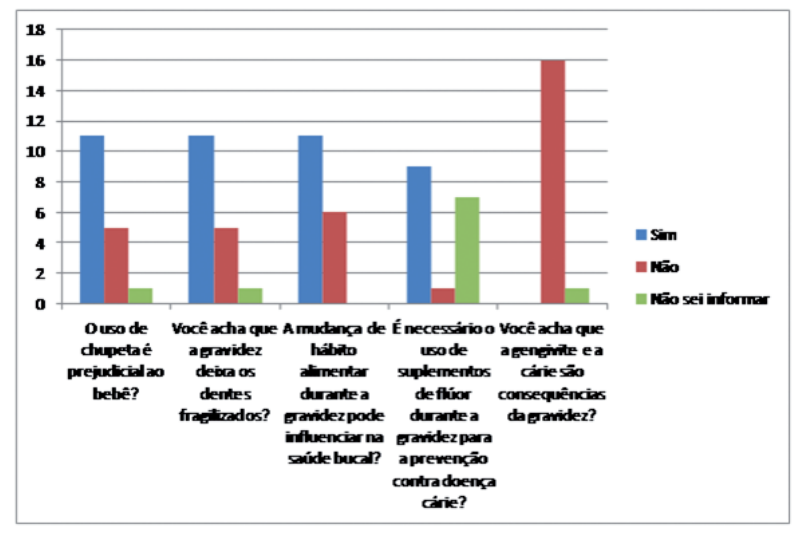

Após o esclarecimento individual de cada pergunta feita no questionário inicial através de palestra educativa, foi realizado o questionário pós-teste. Os dados referentes à segunda aplicação do questionário demonstraram que
$2(11,8 \%)$ gestantes responderam não poder se submeter ao exame radiográfico. Uma $(5,9 \%)$ entrevistada afirmou não saber se existe um período específico para a realização de atendimento odontológico. Com relação ao uso de anestésico local, 1 (5,9\%) acreditou não poder fazer uso do mesmo e $4(23,5 \%)$ declararam que não deveriam realizar extração dentária. No que concerne à alteração bucal, houve uma diferença entre as respostas do primeiro e do segundo questionário. Neste, $9(53 \%)$ gestantes relataram ter observado alguma alteração bucal (Figura 3).

Figura 3 - Distribuição numérica das respostas referentes ao conhecimento das gestantes acerca do acompanhamento e tratamento odontológico durante a gravidez, depois da realização da palestra educativa. Ambulatório Docente Assistencial da Bahiana - ADAB, Salvador/BA, 2013 e 2014.

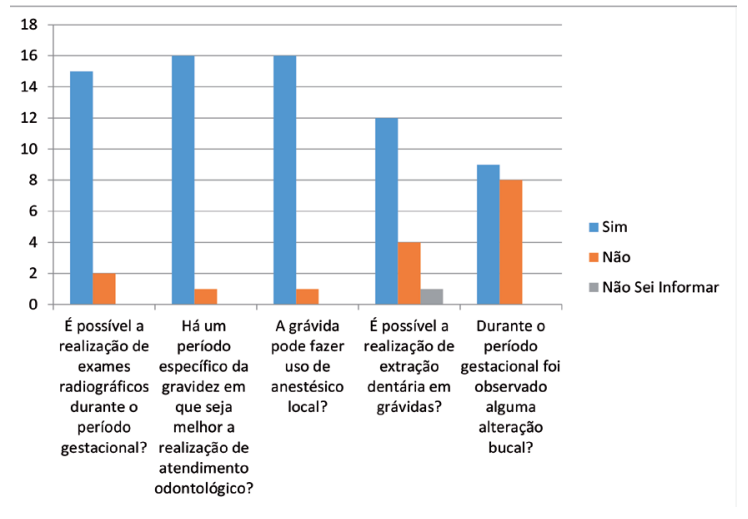

Os resultados referentes às crenças e mitos relacionados à saúde bucal mostraram que 10 (58,8\%) gestantes continuaram a acreditar que a gravidez fragiliza os dentes. Três $(17,7 \%)$ relataram que a suplementação de flúor é necessária neste período, e $13(76,4 \%)$ responderam que o uso de chupeta é prejudicial ao bebê, mas desta vez destacaram a sua relação com a frequência e intensidade do hábito. No que concerne à mudança de hábito alimentar e saúde bucal, $16(94,1 \%)$ entrevistadas responderam haver influência nesta relação (Figura 4).

Figura 4 - Distribuição numérica das respostas referentes às crenças e mitos relacionados à saúde bucal, depois da realização da palestra educativa. Ambulatório Docente Assistencial da Bahiana-ADAB, Salvador/BA, 2013 e 2014.

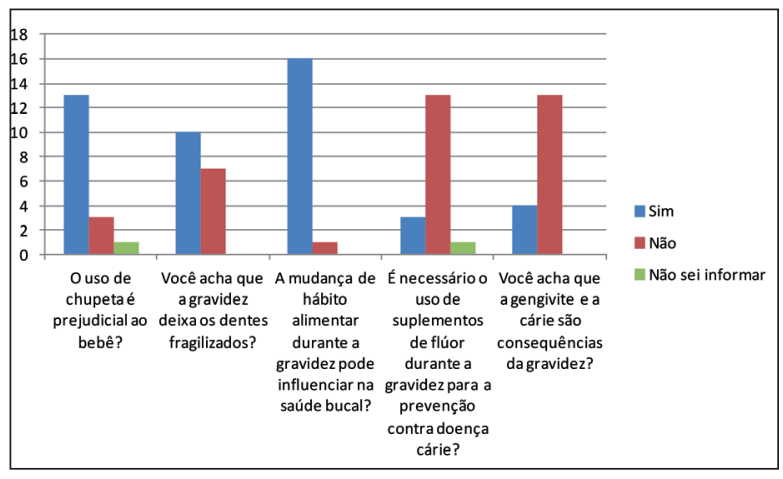

Rev. Ciênc. Méd. Biol., Salvador, v. 15, n. 2, p. 193-198, mai./ago. 2016 


\section{DISCUSSÃO}

O presente estudo parece ter sido o primeiro a avaliar a condição de saúde bucal e as atitudes frente à higiene bucal, crenças e mitos relacionados à saúde bucal e ao atendimento odontológico, em gestantes usuárias do serviço público de saúde do Ambulatório Docente Assistencial da Bahiana - ADAB, Salvador/BA.

Dentre as 17 entrevistadas, a maioria apresentou idade superior a 20 anos e renda familiar superior a um salário mínimo, resultado semelhante ao estudo de Rosa et al. (2007). Mais da metade da amostra concluiu o $2 \circ$ grau. Num estudo realizado por Nogueira et al. (2012) com uma amostra de 200 gestantes, $55 \%$ delas possuíam o ensino superior completo. Mesmo assim, mais da metade delas (57\%) recusaram o tratamento odontológico durante a gestação. Logo, conclui-se que a desinformação não é exclusividade das gestantes com grau de educação formal baixo.

A avaliação da condição gengival das gestantes demonstrou que a maioria delas apresentou Índice de Placa (IP) mais elevado que o Índice Gengival (IG). Num estudo realizado em 2004 por Lima et al. (2005), os autores observaram que todas as pacientes apresentavam Índice Gengival maior que o Índice de Placa. Contudo, ambos os estudos constataram em seus resultados a presença de inflamação gengival durante o período da gravidez e evidenciaram que $100 \%$ das gestantes examinadas apresentavam gengivite.

Foi constatada, no presente estudo, presença de alterações gengivais, sendo altos os índices coletados. Dentre as gestantes examinadas, $64,7 \%$ relataram ter observado alguma alteração gengival, a exemplo de sangramento durante a escovação e gengiva edemaciada. Este resultado é semelhante aos descritos no estudo de Alves et al. (2010) (59,3\%) e Moimaz et al. (2006) $(67,06 \%)$, os quais demonstraram que a presença de sangramento foi um dos problemas frequentemente relacionados à gestação.

Embora na gravidez as alterações hormonais repercutam na fisiologia bucal modificando o equilíbrio normal e possam levar à exacerbação das afecções gengivais, não é o período gestacional por si só o responsável por tais alterações. A gravidez pode afetar a severidade das áreas previamente inflamadas e acentuar a resposta gengival para a placa, modificando o quadro clínico periodontal (ROSA et al., 2007). A maioria das enfermidades gengivais que coincide com a gravidez pode ser tratada mediante a eliminação dos fatores locais, procedendo-se à higiene bucal cuidadosa e ao controle periódico pelo cirurgião-dentista, que deverá motivar a gestante para o comportamento preventivo(REIS et al., 2010). Neste sentido, o tratamento periodontal deve ser incentivado nos programas de saúde pública a fim de promover melhorias no atendimento direcionado às gestantes (ALVES et al., 2010).

A avaliação de atitudes frente aos diversos aspectos de saúde bucal pode contribuir de forma considerável na identificação dos determinantes comportamentais que causam doenças bucais, visto que a saúde bucal é altamente dependente do comportamento do indivíduo (ALVES et al., 2010). Estas informações podem contribuir para a criação de programas educativos e preventivos que motivam a mudança de hábitos frente à saúde (REIS et al., 2010). As atitudes das gestantes foram avaliadas por meio de um questionário constituído de 18 perguntas, aplicado duas vezes, totalizando 36 perguntas. Destas, 10 foram analisadas. Os dados referentes à primeira aplicação do questionário demonstraram que para $47 \%$ das gestantes, não há um período específico da gravidez em que seja meIhor a realização de atendimento odontológico. Entretanto, de acordo com o Ministério da Saúde em 2008 (BRASIL, 2008), há um período preferencial para a realização de intervenções clínicas e procedimentos odontológicos que é o segundo trimestre da gestação. Em 2010, o Ministério da Saúde publicou informações adicionais no Manual Técnico de Gestação de Alto Risco, afirmando que as grávidas poderiam ser tratadas pelo cirurgião-dentista em qualquer período gestacional. Aquelas cuja gravidez tivesse um curso normal e cujo tratamento envolvesse apenas prevenção, profilaxia e restaurações simples, deveriam ser atendidas nas Unidades Básicas de Saúde (UBS). As gestantes com alterações sistêmicas não controladas deveriam ser encaminhadas para Centros de Atendimentos Especializados em Odontologia, a exemplo dos Centros de Especialidades Odontológicas (CEO) (BRASIL, 2010). Todavia, na prática clínica, muitas vezes as gestantes desconhecem os serviços de saúde aos quais devem se dirigir a fim de realizar o seu tratamento odontológico e o julgam necessário apenas quando apresentam processos patológicos em caráter de urgência.

Em concordância com os resultados encontrados por Trevisan e Pinto (2013), o presente trabalho constatou que algumas gestantes demonstraram insegurança sobre quais tipos de intervenções odontológicas são possíveis nesse período, ao mesmo tempo em que apontaram a crença em restrição a pelo menos um tipo de tratamento odontológico durante a gestação, dentre os quais destacam-se o risco das anestesias, do uso de raios $x$ e o risco de exodontias causarem hemorragias, prejudicando o bebê.

O presente estudo confirmou a existência de crenças e mitos acerca do tratamento odontológico, uma vez que a maioria das entrevistadas, na primeira aplicação do questionário, concordou que extração dentária $(64,7 \%)$, uso de anestésico local (53\%) e exames radiográficos (41,2\%) constituem-se proibições durante todo o período gestacional. Esses dados corroboram o estudo de Alves et al. (2010), no qual mais da metade das gestantes entrevistadas afirmou estar proibida a realização destes procedimentos durante a gravidez.

Na população estudada, $64,7 \%$ das gestantes acharam que era normal desenvolver cárie dentária durante o período gestacional, uma vez que muitas mães relacionaram que os dentes ficam mais fracos pela transmissão de minerais, como o cálcio, para os dentes do bebê. Bastiani et al. (2010) relataram em sua pesquisa que $48,75 \%$ das entrevistadas também fizeram esta associação. No estudo de Mesquita et al. (2013), as gestantes indicaram a gravidez como fator para o desenvolvimento da doença cárie e perda dentária, 
destacando assim o folclore popular como rico em atributos negativos em relação à saúde bucal na gravidez.

Apesar de atualmente os mitos relacionados à cárie dentária e fragilidade dos dentes na gestação estarem menos dissipados na sociedade, ainda existem crenças errôneas, como "a cada gravidez se perde um dente" que devem ser esclarecidas, salientando o fato de que os principais motivos para ocorrência da cárie e perda dentária são a negligência com a higiene bucal e a falta de cuidados com os hábitos alimentares (BASTIANI et al., 2010).

Quando questionadas sobre a influência que a mudança do hábito alimentar pode ter sobre a saúde bucal, $35,3 \%$ das gestantes afirmaram não haver influência. Entretanto, segundo Moimaz et al. (2006), a higiene bucal deficiente e o acúmulo de placa, muitas vezes exacerbado durante a gestação pelo aumento da frequência de ingestão de alimentos açucarados e o descuido inversamente proporcional na higienização, aumentam a possibilidade de desenvolvimento da doença periodontal.

Após a primeira aplicação do questionário, foram feitos esclarecimentos acerca de cada pergunta e o mesmo questionário foi reaplicado. $\mathrm{Na}$ alternativa que perquiria às gestantes se a mudança de hábito alimentar influenciava na saúde bucal, 94,1\% delas afirmaram que sim. Os dados referentes à segunda aplicação do questionário demonstraram que a informação é essencial para mudança de hábitos na saúde bucal, e quando esta é transmitida por profissionais de saúde, há uma maior credibilidade e segurança (REIS et al., 2010).

Sendo assim, urge a necessidade de desenvolvimento de políticas públicas com medidas educativo-preventivas voltadas para esse grupo especial de mulheres, visto que as gestantes constituem um grupo populacional estratégico em saúde. Nesta fase, elas se encontram mais receptivas à aquisição de novos conhecimentos e estão dispostas à mudança de hábitos para beneficiar seu bebê (REIS et al., 2010). Todavia, essa educação deve ser realizada de forma multidisciplinar pelos profissionais de saúde (ginecologista, obstetra, psicólogo) com o objetivo de ampliar o conhecimento das gestantes. À esta equipe, some-se a presença de um cirurgião-dentista como agente promotor de saúde durante o período pré-natal.

\section{CONCLUSÃO}

$O$ presente estudo sugere fortemente que $\mathrm{o}$ atendimento odontológico no período pré-natal é de suma importância para prevenção e controle de afecções que podem surgir ou serem agravadas durante a gestação, em razão das inúmeras alterações ocorridas no organismo deste grupo populacional. De fato, mitos e crenças populares ainda exercem um impacto negativo no teor das informações das gestantes, afastando-as do tratamento odontológico. Todavia, a realização de ações de cunho educativo mostrou-se eficaz e capaz de mudar essa realidade.

\section{REFERÊNCIAS}

1. AINAMO, J.; BAY, I. Problems and proposals for recording gengivitis and plaque. Int. dent. j., London, v. 25, n. 4, p. 229-235, Dec. 1975.

2. Albuquerque, O. M. R.; ABEGG, C.; ROdRIGUES C. S. Percepção de gestantes do Programa Saúde da Família em relação a barreiras no atendimento odontológico em Pernambuco, Brasil. Cad. saúde pública, Rio de Janeiro, v. 20, n. 3, p. 789-796, jun. 2004.

3. ALVES, R. T. et al. Perfil epidemiológico e atitudinal de saúde bucal de gestantes usuárias do serviço público de Juiz de Fora, MG. Pesq. Bras. Odontoped. Clin. Integr., João Pessoa, v. 10, n. 3, p. 413-421, set./dez. 2010.

4. BASTIANI, C. et. al. Conhecimento das gestantes sobre alterações bucais e tratamento odontológico durante a gravidez. Odontol. clín.-cient., Recife, v. 9, n. 2, p. 155-160, abr./jun. 2010.

5. BRASIL. Ministério da Saúde. Secretaria de Atenção à Saúde. Departamento de Ações Programáticas Estratégicas. Gestação de alto risco: manual técnico. 5 ed. Brasília: Ministério da Saúde, 2010. (Série A, Normas e Manuais Técnicos).

6. BRASIL. Ministério da Saúde. Secretaria de Atenção à Saúde. Departamento de Atenção Básica. Saúde Bucal. Cadernos de Atenção. Brasília: Ministério da Saúde, 2008. (Série A, Normas e Manuais Técnicos, 17).

7. DE CAMARGO, E. C. ; SOIBELMAN, M. Prevalência da doença periodontal na gravidez e sua influência na saúde do recém-nascido. Rev. AMRIGS, Porto Alegre, v. 49, n. 1, p. 11-15, jan./mar. 2005.

8. GRANDI, C. ; MERITANO, J. ; TRUNGADI, M. Doença periodontal materna e parto pré-termo: um estudo de caso-controle. Rev. Pan-Amazônica Saúde, Buenos Aires, v. 1, n. 2, p. 41-48, mar. 2010.

9. LIMA, L. L. et. al. Levantamento em saúde bucal de gestantes de São Luís-MA: análise da condição periodontal e necessidade de tratamento. Rev. Int. Periodontia Clin., Maranhão, v. 2, n. 4, p.17-22, 2005.

10. MESQUITA, B. S. et. al. Aspectos Norteadores da Atenção à Saúde Bucal de Gestantes da Estratégia Saúde da Família de João Pessoa, Brasil. Rev. Odontol. Bras. Central, Goiânia, v. 21, n. 60, p. 45-49, 2013.

11. MOIMAZ, S. A. S. et. al. Condição periodontal durante a gestação em um grupo de mulheres brasileiras. Ciênc. odontol. bras., São José dos Campos, v. 9, n. 4, p. 59-66, out./dez. 2006.

12. NOGUEIRA, L. T. et. al. Retardo na procura do tratamento odontológico e percepção da saúde bucal em mulheres grávidas. Odontol. clín.-cient., Recife, v. 11, n. 2, p. 127-131, abr./jun. 2012.

13. REIS, D. M. et. al. Educação em saúde como estratégia de promoção de saúde bucal em gestantes. Ciênc. saúde coletiva, Rio de Janeiro, v. 15, n. 1, p. 269-276, 2010.

14. ROSA, P. C. et. al. Indicadores de saúde bucal de gestantes vinculadas ao programa de pré-natal em duas unidades básicas de saúde em Porto Alegre/RS. Arq. odontol., Belo Horizonte, v. 47, n. 1, p. 36-43, 2007.

15. TREVISAN, C. L.; PINTO, A. A. M. Fatores que Interferem no Acesso e na Adesão das Gestantes ao Tratamento Odontológico. Arch. health invest., Araçatuba, v. 2, n. 2, p. 29-35, 2013.

16. VASCONCELOS, R. G. et. al. Atendimento odontológico a pacientes gestantes: como proceder com segurança. Rev. bras. odontol., Rio de Janeiro, v. 69, n. 1, p. 120-124, jan./jun.2012.

Submetido em: 23/09/2015

Aceito em: 15/04/2016 